\title{
Initial state in heavy ion collisions
}

\author{
T. Lappi
}

Department of Physics, P.O. Box 35, 40014 University of Jyväskylä, Finland and Helsinki Institute of Physics, P.O. Box 64, 00014 University of Helsinki, Finland

\begin{abstract}
We briefly review advances in understanding the initial stages of a heavy ion collision. In particular the focus is on moving from parametrizing the initial state to calculating its properties from QCD, consistently with the description of hard probes and dilute-dense scattering experiments. Modeling the event-by-event fluctuating nuclear geometry in initial state calculations has significantly improved in recent years. We also discuss prospects of directly seeing effects of particle correlations created in the initial state in the experimental observables.
\end{abstract}

Keywords:

\section{Introduction}

For a practitioner of hydrodynamic modeling of heavy ion collisions, the question of initial conditions often boils down to an acronym soup of models. One tries out a suitable subset of these ("Glauber", KLN, [mc]KLN, mcrcBK, EPOS, EKRT, IPglasma etc.) as an input to a fluid dynamics calculation and compares to experimental data. The purpose here is very different, namely to discuss the physics ingredients in these calculations and concentrate more on their common aspects them than on the differences. This is done with the caveat that we are concentrating exclusively on a weak coupling partonic description of the degrees of freedom involved. We will start with a very brief introduction to the physics picture in the weak coupling approach. We then discuss dilute-dense control experiments that can be used to more directly probe the degrees of freedom in the initial state of a heavy ion collision. The most recent dynamical initial state models will then be discussed in comparison to Monte Carlo Glauber parametrizations. Finally we will describe recent calculations of long range correlations that, if one is able to discriminate between effects of collective flow and those originating from the initial state, could provide a direct access to the latter.

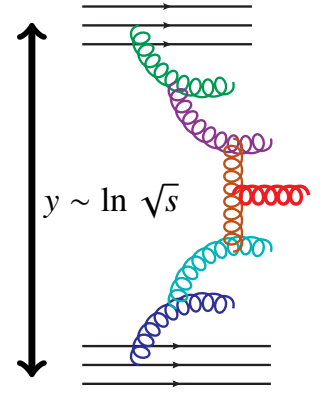

Figure 1: Gluon cascade leading to particle production in the central rapidity region.

\section{Initial state at weak coupling}

The weak coupling picture of particle production at central rapidities in very high energy hadronic collisions is depicted in Fig. 1. The gluons that are mainly responsible for particle production result from a cascade of multiple splittings from the initial valence quarks. The emission probability for one splitting is $\alpha_{\mathrm{s}} \mathrm{d} x / x$, where $x$ is the fraction of the longitudinal momentum of the incoming hadron and $\alpha_{\mathrm{s}}$ is the QCD coupling. This form is constant as a function of rapidity, and thus leads naturally to a rapidity plateau, i.e. a multiplicity $\mathrm{d} N / \mathrm{d} y$ 
approximately independent of $y$ for scales $\Delta y \lesssim 1 / \alpha_{\mathrm{s}}$. While at RHIC the total collision energy is still so low that the $y$-dependence is dominated by large- $x$ physics, at the LHC this plateau has become prominently visible in the experimental data. At high enough energy there is phase space for many gluon emissions in the cascade, each additional one suppressed by a factor $\alpha_{\mathrm{s}}$ but enhanced by a phase space volume $\Delta y \sim \ln \sqrt{s}$. With a factor $1 / n$ ! from the rapidity ordering of $n$ gluons one could in fact very roughly expect

$$
\frac{\mathrm{d} N_{g}}{\mathrm{~d} y} \sim \sum_{n} \frac{1}{n !}\left(\alpha_{\mathrm{s}} \ln \sqrt{s}^{n} \sim \sqrt{s}^{\alpha_{\mathrm{s}}}\right.
$$

gluons. Again, the experimental energy dependence of multiplicities in pp $\left(\sim \sqrt{s}^{0.2}\right)$ and AA $\left(\sim \sqrt{s}^{0.3}\right)$ collisions fits in very nicely with this very crude weak coupling, small angle scattering estimate. This could be contrasted with a strong coupling picture, which would generically lead to complete baryon stopping [1] and a stronger growth of the multiplicity with $\sqrt{s}$.

Eventually the gluons in this cascade will overlap and gluon mergings will start to compete with splittings. This increase in the gluon density corresponds to the Yang-Mills theory becoming completely nonperturbative. This happens when the two terms of the covariant derivative $-i D_{\mu}=-i \partial_{\mu}+g A_{\mu}=p_{\mu}+g A_{\mu}$ are of the same order. The (transverse) momentum scale at which this happens is referred to as the saturation scale $p_{T} \sim g A_{\perp} \sim Q_{\mathrm{s}}$. When the energy is high enough, $Q_{\text {s }} \gg \Lambda_{\mathrm{QCD}}$ and the coupling is still weak. What results is a picture that has a weak coupling $g$ but is still nonperturbative due to the large gluon field strengths $A_{\mu} \sim 1 / g$. Since the occupation numbers of gluonic states $f(k) \sim A_{\mu} A_{\mu} \sim 1 / \alpha_{\mathrm{s}}$ are large, the gluon field is, to leading order in the coupling, a classical field.

Our understanding of how exactly such a system of strong (but very anisotropic) gluon fields thermalizes (or slightly more modestly isotropizes) has seen significant progress in the recent years (for a review see [2]). Pursuing this question in any depth is out of scope here, but as a rough outline the process must behave in the following way. The initial classical fields, with with occupation numbers $f(k) \sim \frac{1}{\alpha_{s}} \gg 1$ are very anisotropic due to the longitudinal expansion of the system. As gluons start to scatter off the transverse plane and occupy a larger volume in phase space, the occupation number decreases. When $f(k) \ll \frac{1}{\alpha_{\mathrm{s}}}$ one can switch to a kinetic theory description and follow the system towards local equilibrium. Close to isotropy kinetic theory matches smoothly into a $2^{\text {nd }}$ order viscous hydrodynamical description. A recent weak coupling calculation using an

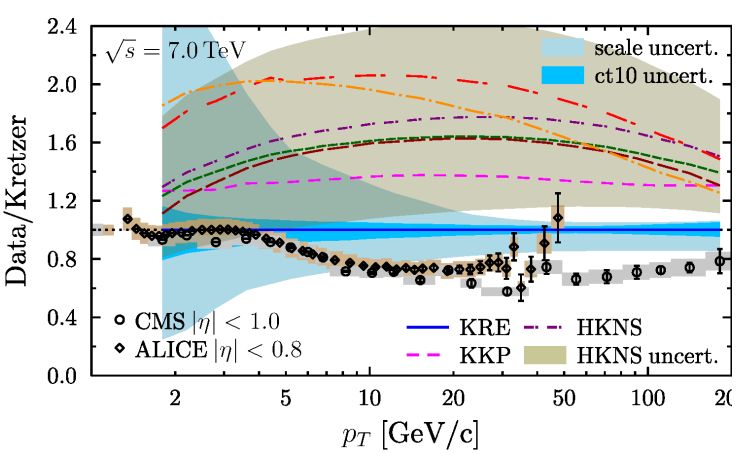

Figure 2: Different fragmentation functions in NLO pQCD compared to CMS data for charged particle spectra in proton-proton collisions, from [9].

effective kinetic theory for QCD [3] arrives, when extrapolating to realistic values of $\alpha_{\mathrm{s}}$, to times as short as $1 \mathrm{fm}$ for a matching to viscous hydrodynamics.

\section{Dilute-dense control measurements}

The quintessential experiment for measuring the partonic content of a hadron or nucleus is deep inelastic scattering (DIS). A good description of the initial state of a heavy ion collision should be consistent with the precise measurements of quarks and gluons in a proton at HERA and with the available electron-nucleus cross section data. It should also be able to give quantitative predictions for future measurements at an EIC. This is naturally true for calculations whose starting point are nuclear pdfs (e.g. EPS09 [4]). It also applies to more recent CGC calculations using e.g. the IPSat or bCGC parametrizations $[5,6]$ and to calculations using the running coupling Balitsky-Kovchegov equation (rcBK), e.g. [7, 8]. In the classical field CGC picture the initial color fields in a heavy collision are calculated in terms of Wilson lines, whose correlator determines the total DIS cross section.

Another check of QCD descriptions is provided by hadronic dilute-dense, i.e. proton-nucleus, collisions. The most straightforward observables here are ratios of particle spectra in proton-nucleus collisions to those in proton-proton ones, normalized with the number of nucleons in the nucleus, known as $R_{p A}$. At high $p_{T}$ these ratios would be expected to approach unity for many particle species. This is particularly important when moving from minimum bias proton-nucleus collisions to separate centrality classes, where hard prticle production serves as an important consistency check of the Glauber modeling [10] needed for the centrality determination. If the normalization of $R_{p A}$ is under control, 
its small- $p_{T}$ behavior is sensitive to the enhancement of saturation effects in a nucleus, especially at forward rapidity where one probes smaller values of $x$ in the nucleus.

Typical CGC calculations of hadronic $R_{p A}$ at semihard $p_{T}$ use two different formalisms. The forward rapidity "hybrid" picture starts from a collinear quark or gluon from the probe, which is described in terms of conventional DGLAP evolved parton distribution functions. This parton propagates through the dense color field of the target, picking up an eikonal Wilson line. The other possible treatment is a symmetric $k_{T^{-}}$ factorized description, where the produced gluon spectum is obtained as a convolution of two unintegrated gluon distributions (ugd's) describing the projectile and the target. The ugd is, again, obtained as a Fouriertransform of the Wilson line correlator probed in DIS. Both formalisms require, unexceptionally for leading order QCD calculations, a " $K$-factor" of order $\sim 2$ to fit the spectra, but this normalization uncertainty cancels in the ratio $R_{p A}$. Unlike in the early days of RHIC, up to date calculations of $R_{p A}[11,12,13,8]$ use ugd's that have been fit to the precise HERA data. In fact, because this data is very constraining, the main quantitative difference between these calculations is the treatment of the nuclear geometry needed to go from a proton to a nuclear target. With $R_{p A}$ predictions differing, e.g. by a factor $\sim 2$ from Ref. [11] to [8], this can be a significant effect. A similar example of the importance of a proper treatment of the nuclear geometry is provided by forward $J / \Psi$ production [14].

Even more discriminative power is in principle provided by the absolute spectra themselves. In a weak coupling QCD calculation the normalization has, however, much more uncertainty than the ratio $R_{p A}$. An additional complication comes from the recent observation [9] that current fragmentation functions do not provide a very good description of data (see Fig. 2) even in the cases where they should work well, i.e. protonproton collisions at higher $p_{T}$. This will require further development, focusing in particular on the poorly constrained gluon fragmentation funtions, see e.g. [15]. Electroweak probes (photons and electroweak bosons) are theoretically the cleanest observable probing weak coupling partonic physics in the nucleus. Here the greater question is whether the experimental accuracy will be good enough to distinguish nuclear effects.

\section{MC Glauber and dynamical models}

Initial matter formation in heavy ion collisions is often parametrized in terms of a "Monte Carlo Glauber"
(MCG) model [16]. One starts from nucleons distributed inside the nucleus according to a standard Woods-Saxon nuclear density profile. Nucleons from the colliding projectiles are then deemed to "collide" or "participate" if they lie within a short enough distance (determined by the total inelastic nucleon-nucleon cross section) from each other. Experimental event-by event distributions of charged particle multiplicities or forward calorimeter energies can be roughly modeled by assuming that the multiplicity or energy is proportional to the number of "participant nucleons" or "binary nucleon-nucleon collisions". This has led to the MCG model being used both experimentally in event centrality classification, and theoretically as a spatial distribution of the initial conditions of hydrodynamical simulations.

In hydrodynamical calculations microscopical models of particle production are sometimes contrasted with MCG based on comparisons to experimental data. We would like to argue that this is a fundamentally misleading and unfair comparison. In addition to not being completely unique (one can e.g. choose the multiplicity or the energy as proportional to either $N_{\text {coll }}$ or $N_{\text {part }}$ ), the MCG model is a purely empirical observation without any dynamical mechanism for particle production. It does not, for example, make any predictions about rapidity or $\sqrt{s}$ dependence. The MCG model is such a successful description of nuclear geometry that a similar picture is in fact built into most modern initial state calculations which combine it with a microscopical description of particle production (strings, classical fields, parton scattering ...). Thus it is not surprising that, to a first approximation, the centrality dependence of bulk observables from these models is close to MCG. On a conceptual level, if one is interested in understanding microscopical QCD dynamics, the meaningful comparison is between different dynamical models of particle production, not between them and the MCG. One can ask whether the theory is consistent within itself, or how well the (necessarily small) deviations from a $\mathrm{d} N_{\text {ch }} / \mathrm{d} \eta \sim N_{\text {part }}$ scaling that it predicts describe experimental observations.

Let us recall some examples of how a very similar treatment of the nuclear geometry is at the heart of many recent initial state calculations. The MCKLN model [17] explicitly starts from an MCG calculation that determines, event-by-event, a set of participant nucleons in each nucleus. One then takes the (now probedependent, i.e. nonuniversal) saturation scales in the nuclei as proportional to this $N_{\text {part }}$ and uses them as inputs in a $k_{T}$-factorized calculation of the initial energy density. The MCrcBK model [18] is otherwise similar, but 
the ad hoc functional form of the ugd is replaced by a solution of the BK equation. The IPglasma initial state model [19] starts from the IPsat [20] parametrization for the dipole scattering amplitude in a nucleus

$$
\mathcal{N}_{q \bar{q}}=1-e^{-\frac{\pi^{2}}{2 N_{\mathrm{c}}} \alpha_{\mathrm{s}}\left(\mu^{2}\right) x g\left(x, \mu^{2}\right) \sum_{i=1}^{A} T_{p}\left(\mathbf{b}_{T}-\mathbf{b}_{T i}\right) \mathbf{r}_{T}^{2}},
$$

involving fluctuating nucleon positions $\mathbf{b}_{T i}$ from a Woods-Saxon distribution. One then determines a local saturation scale $Q_{\mathrm{S}}\left(\mathbf{b}_{T}\right)$ from this amplitude and uses it in an MV-model correlator of color charges

$$
\left\langle\rho^{a}\left(\mathbf{x}_{T}\right) \rho^{b}\left(\mathbf{y}_{T}\right)\right\rangle \sim Q_{\mathrm{s}}^{2}\left(\mathbf{x}_{T}\right) \delta^{a b} \delta^{(2)}\left(\mathbf{x}_{T}-\mathbf{y}_{T}\right),
$$

from which the classical Yang-Mills (CYM) field is calculated. The EKRT model in its present version [21] uses a fluctuating nuclear thickness profile

$$
T_{A}\left(\mathbf{b}_{T}\right)=\sum_{i=1}^{A} T_{p}\left(\mathbf{b}_{T}-\mathbf{b}_{T i}\right)
$$

to determine the initial energy density as

$$
\varepsilon\left(\mathbf{b}_{T}\right)=\mathcal{F}\left[T_{A}\left(\mathbf{b}_{T}\right) T_{B}\left(\mathbf{b}_{T}\right)\right],
$$

where the QCD dynamics in the calculation is parametrized by the function $\mathcal{F}$. Also string-based models such as EPOS [22] and AMPT [23] share the same description of the nuclear geometry. Since we are still far from being able to calculate nuclear structure from first principles QCD, at some level the MCG geometry has to be put in by hand, no matter the sophistication of the QCD calculation

Besides the spatial, distribution the calculation of the initial state should naturally also provide the value of the energy (or entropy) density. Ideally this should be done without any adjustable free parameters, but as we will now discuss this is not fully the case for any of the currently available calculations. In a purely perturbative calculation the initial gluon multiplicity is not finite, so the question of normalization is intimately tied in with the issue of infrared cutoffs. This is a particularly salient feature of the EKRT model [24], where the initial parton spectrum calculated in collinear perturbation theory has a strong power law dependence on the infrared cutoff. The model introduces two essential free parameters, the cutoff (saturation scale) $p_{0}$ and a normalization coefficient $K_{\text {sat }}$, which are simultaneously determined from the final state saturation criterion

$$
\frac{\mathrm{d} E_{T}\left(p_{T}>p_{0}\right)}{\mathrm{d}^{2} \mathbf{x}_{T}}=K_{\text {sat }} \frac{p_{0}^{3} \Delta y}{\pi}
$$

and by fitting the multiplicity in the most central LHC lead-lead collisions. In KLN-type initial conditions that use a $k_{T}$-factorized formula to compute gluon production the procedure is slightly different. Because of parton saturation the total gluon multiplicity is only logarithmically infrared divergent

$$
\frac{\mathrm{d} N}{\mathrm{~d}^{2} \mathbf{p}_{T} \mathrm{~d} y}=\frac{C}{\alpha_{\mathrm{s}}} \frac{1}{\mathbf{p}_{T}^{2}} \int_{\mathbf{k}_{T}} \varphi_{y}\left(\mathbf{k}_{T}\right) \varphi_{y}\left(\mathbf{p}_{T}-\mathbf{k}_{T}\right),
$$

where $\varphi_{y}\left(\mathbf{k}_{T}\right)$ is the unintegrated gluon distribution. Thus, in stead of determining the cutoff together with the normalization, one usually chooses an infrared cutoff scale (often by restricting the $\mathbf{k}_{T}$-integral to $k_{T} \leq p_{T}$; for a different scheme see [12]) and then adjusts the normalization constant $C$ to data. This leaves the gluon mean $p_{T}$ (energy per particle) quite unconstrained and potentially very far from a value extrapolated backward from the experimental data [18].

In the CYM calculations, such as the IPglasma model [19], the gluon multiplicity is finite due to nonlinear effects in the final state [25] and no infrared cutoffs are needed. The Wilson lines $U\left(\mathbf{x}_{T}\right)$ that fully determine the initial conditions of the CYM calculation [26] are constrained by DIS data because the correlator

$$
\frac{1}{N_{\mathrm{c}}} \operatorname{Tr} U^{\dagger}\left(\mathbf{x}_{T}\right) U\left(\mathbf{y}_{T}\right)
$$

is proportional to the total DIS cross section. Thus on a conceptual level there are no free adjustable parameters in the CYM scenario. The practical implementation in IPglasma, however, uses a procedure that leaves room for small uncertainties. One first uses a fit to HERA data to get the saturation scale $Q_{\mathrm{s}}^{2}\left(\mathbf{b}_{T}\right)$. This is then input into an MV model calculation so that one reproduces the same $Q_{\mathrm{s}}$, but not the full Wilson line correlator of the DIS fit. Additionally, in the MV model in a finite nucleus one must regulate long distance Coulomb tails of the classical gauge fields with a confinement scale parameter. The net result of this procedure is that the Wilson line in the CYM initial condition does not exactly match the original DIS cross section, although it is very well constrained by it.

\section{Correlations: direct signals of the initial state?}

A simple causality argument shows that correlations in particle production that have a long range in rapidity must have originated in the earliest stage of the collision process. This is analogous to the way correlations in the cosmic microwave background are sensitive to early times, when now separate parts of the universe were still in causal contact. Azimuthal flow coefficients $v_{n}$ are an important example of long range rapidity correlations. 


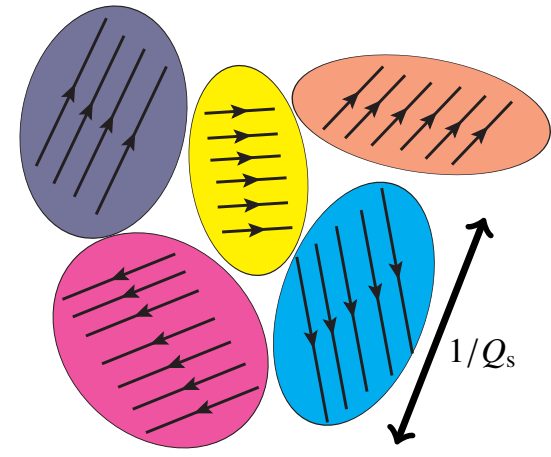

Figure 3: Color field domains in the target

They are either explicitly constructed from a (usually rapidity separated) multiparticle correlation function (the "cumulant method") or by correlating particles at one rapidity with a reaction plane determined from particles in another part of the detector. The same correlation can be presented in terms of a yield per trigger or as a flow coefficient $v_{n}$; see e.g. [27]. The most important origin of these correlations, especially in large collision systems, is the geometry of the initial state: the distribution of matter in the transverse plane is by definition felt at all rapidities. Geometrical correlations are, however, in position space, and strong enough collective final state interactions are needed to transform them into observable momentum space distributions.

It has more recently [28, 29] been pointed out that, in particular in small collision systems, the domain structure in the target color field can also generate azimuthal correlations directly in momentum space without final state collective phenomena. These correlations follow in a very intuitive way from the CGC picture. The usual picture of particle production in this case is that of an individual quark or gluon (whose number is given by a conventional pdf) passing through the strong color field of the target and being deflected to some transverse momentum. Since the target color field consists of domains of size $\sim 1 / Q_{\mathrm{s}}^{2}$ (see Fig. 3), incoming particles in the same color state and hitting the same domain experience a similar deflecting color field and become correlated. The mechanism generates correlations that are suppressed by the number of independent domains and colors as $\sim 1 /\left(N_{\mathrm{c}}^{2} Q_{\mathrm{s}}^{2} S_{\perp}\right)$, where $S_{\perp}$ is the size of interaction area. Thus, in contrast to collective flow effects, these correlations are enhanced in small collision systems.

These correlations have recently been analyzed by different authors in calculations that share the same physical picture, but differ in the approximations used to calculate the correlations of the target color fields. The "Glasma graph" calculations [28, 30] linearize in the color charge density of the target and assume Gaussian correlations between the domains. In the "color field domain model" [31] one also linearizes in the target field, but adds an additional non-Gaussian correlation. On the other hand one can perform a full nonlinear calculation in the dilute-dense limit $[32,33]$ without adding non-Gaussian correlations. By performing a full CYM simulation [34] one can also include preequilibrium collective effects in the calculation of the azimuthal dependence. For a more detailed reecnt discussion of the differences and similarities see [35]. So far all of these calculations assume parametrically small rapidity separations $\Delta y \lesssim 1 / \alpha_{\mathrm{s}}$, but there have been proposals to extend the calculation also to parametrically large rapidity intervals [36].

\section{Conclusions}

In conclusion, recent years have seen a huge progress towards a more well defined weak coupling picture of the initial stages of a heavy ion collision. Studies of thermalization are moving from qualitative to quantitative, and the importance of interfacing with kinetic theory for the later stage of equilibration is becoming understood. One wants the initial stage calculation to be consistent with perturbative probes and control measurements in dilute-dense collisions. We emphasized that the MC Glauber-like geometry is built into the more modern dynamical models of the initial state. Finally we discussed the azimuthal structure of long range rapidity correlations, i.e. the $v_{n}$-coefficients, especially in small systems, where the interplay between initial and final state collective effects remains to be quantitatively sorted out.

This work has been supported by the Academy of Finland, projects 267321 and 273464.

\section{References}

[1] Y. V. Kovchegov, Early time dynamics in heavy ion collisions from CGC and from AdS/CFT, Nucl. Phys. A830 (2009) 395c402c. arXiv:0907.4938, doi:10.1016/j.nuclphysa. 2009.09.035.

[2] F. Gelis, Initial state and thermalization in the color glass condensate framework, in: X. N. Wang (Ed.), Quark gluon plasma 5, World Scientific, 2015. arXiv:1508.07974, doi: 10.1142/S0218301315300088.

[3] A. Kurkela, Y. Zhu, Isotropization and hydrodynamization in weakly coupled heavy-ion collisionsarXiv: 1506.06647.

[4] K. J. Eskola, H. Paukkunen, C. A. Salgado, EPS09 - a new generation of NLO and LO nuclear parton distribution functions, JHEP 04 (2009) 065. arXiv:0902.4154, doi:10.1088/ 1126-6708/2009/04/065. 
[5] A. H. Rezaeian, M. Siddikov, M. Van de Klundert, R. Venugopalan, Analysis of combined HERA data in the impactparameter dependent saturation model, Phys. Rev. D87 (2013) 034002. arXiv:1212.2974, doi:10.1103/PhysRevD.87. 034002.

[6] A. H. Rezaeian, I. Schmidt, Impact-parameter dependent color glass condensate dipole model and new combined HERA data, Phys. Rev. D88 (2013) 074016. arXiv:1307.0825, doi: 10 . 1103/PhysRevD.88.074016.

[7] J. L. Albacete, N. Armesto, J. G. Milhano, P. Quiroga-Arias, C. A. Salgado, AAMQS: a non-linear QCD analysis of new HERA data at small-x including heavy quarks, Eur. Phys. J. C71 (2011) 1705. arXiv:1012.4408, doi:10.1140/epjc/ s10052-011-1705-3.

[8] T. Lappi, H. Mäntysaari, Single inclusive particle production at high energy from HERA data to proton-nucleus collisions, Phys. Rev. D88 (2013) 114020. arXiv: 1309.6963, doi:10.1103/ PhysRevD.88.114020.

[9] D. d'Enterria, K. J. Eskola, I. Helenius, H. Paukkunen, Confronting current NLO parton fragmentation functions with inclusive charged-particle spectra at hadron colliders, Nucl. Phys. B883 (2014) 615-628. arXiv:1311.1415, doi:10.1016/j. nuclphysb.2014.04.006.

[10] J. Adam, et al., Centrality dependence of particle production in p-Pb collisions at $\sqrt{s_{\mathrm{NN}}}=5.02 \mathrm{TeV}$, Phys. Rev. C91 (2015) 064905. arXiv:1412.6828, doi:10.1103/PhysRevC.91. 064905.

[11] J. L. Albacete, C. Marquet, Single inclusive hadron production at RHIC and the LHC from the color glass condensate, Phys. Lett. B687 (2010) 174-179. arXiv:1001.1378, doi: 10.1016/j.physletb.2010.02.073

[12] P. Tribedy, R. Venugopalan, QCD saturation at the LHC: comparisons of models to $\mathrm{p}+\mathrm{p}$ and $\mathrm{A}+\mathrm{A}$ data and predictions for $\mathrm{p}+\mathrm{Pb}$ collisions, Phys. Lett. B710 (2012) 125-133. arXiv: 1112.2445, doi:10.1016/j.physletb.2012.02.047.

[13] A. H. Rezaeian, CGC predictions for $\mathrm{p}+\mathrm{A}$ collisions at the LHC and signature of QCD saturation, Phys. Lett. B718 (2013) 1058-1069. arXiv:1210.2385, doi:10.1016/j. physletb.2012.11.066.

[14] B. Ducloué, T. Lappi, H. Mäntysaari, Forward $J / \psi$ production in proton-nucleus collisions at high energy, Phys. Rev. D91 (11) (2015) 114005. arXiv: 1503.02789, doi : 10.1103/ PhysRevD.91.114005.

[15] D. de Florian, R. Sassot, M. Epele, R. J. Hernández-Pinto, M. Stratmann, Parton-to-pion fragmentation reloaded, Phys. Rev. D91 (1) (2015) 014035. arXiv:1410.6027, doi:10. 1103/PhysRevD.91.014035.

[16] M. L. Miller, K. Reygers, S. J. Sanders, P. Steinberg, Glauber modeling in high energy nuclear collisions, Ann. Rev. Nucl. Part. Sci. 57 (2007) 205-243. arXiv:nucl-ex/0701025, doi:10.1146/annurev.nucl.57.090506.123020.

[17] H. J. Drescher, Y. Nara, Effects of fluctuations on the initial eccentricity from the color glass condensate in heavy ion collisions, Phys. Rev. C75 (2007) 034905. arXiv:nucl-th/ 0611017, doi:10.1103/PhysRevC.75.034905.

[18] J. L. Albacete, A. Dumitru, A model for gluon production in heavy-ion collisions at the LHC with rcBK unintegrated gluon densitiesarXiv: 1011.5161.

[19] B. Schenke, P. Tribedy, R. Venugopalan, Fluctuating glasma initial conditions and flow in heavy ion collisions, Phys. Rev. Lett. 108 (2012) 252301. arXiv:1202.6646, doi:10.1103/ PhysRevLett.108.252301.

[20] H. Kowalski, D. Teaney, An impact parameter dipole saturation model, Phys. Rev. D68 (2003) 114005. arXiv: hep-ph/ 0304189, doi:10.1103/PhysRevD.68.114005.
[21] H. Niemi, K. J. Eskola, R. Paatelainen, Event-by-event fluctuations in perturbative QCD + saturation + hydro model: pinning down QCD matter shear viscosity in ultrarelativistic heavy-ion collisionsarXiv: 1505.02677.

[22] K. Werner, I. Karpenko, T. Pierog, M. Bleicher, K. Mikhailov, Event-by-event simulation of the three-dimensional hydrodynamic evolution from flux tube initial conditions in ultrarelativistic heavy ion collisions, Phys. Rev. C82 (2010) 044904. arXiv:1004.0805, doi:10.1103/PhysRevC.82.044904.

[23] Z.-W. Lin, C. M. Ko, B.-A. Li, B. Zhang, S. Pal, A multiphase transport model for relativistic heavy ion collisions, Phys. Rev. C72 (2005) 064901. arXiv:nucl-th/0411110, doi: 10.1103/PhysRevC.72.064901.

[24] R. Paatelainen, K. J. Eskola, H. Niemi, K. Tuominen, Fluid dynamics with saturated minijet initial conditions in ultrarelativistic heavy-ion collisions, Phys. Lett. B731 (2014) 126130. arXiv:1310.3105, doi:10.1016/j.physletb. 2014. 02.018.

[25] J. P. Blaizot, T. Lappi, Y. Mehtar-Tani, On the gluon spectrum in the glasma, Nucl. Phys. A846 (2010) 63-82. arXiv: 1005. 0955, doi:10.1016/j.nuclphysa.2010.06.009.

[26] A. Kovner, L. D. McLerran, H. Weigert, Gluon production from non-abelian Weizsäcker-Williams fields in nucleus-nucleus collisions, Phys. Rev. D52 (1995) 6231-6237. arXiv:hep-ph/ 9502289, doi:10.1103/PhysRevD.52.6231.

[27] G. Aad, et al., Measurement of long-range pseudorapidity correlations and azimuthal harmonics in $\sqrt{s_{N N}}=5.02 \mathrm{TeV}$ protonlead collisions with the ATLAS detector, Phys. Rev. C90 (2014) 044906. arXiv:1409.1792, doi:10.1103/PhysRevC. 90. 044906.

[28] A. Dumitru, K. Dusling, F. Gelis, J. Jalilian-Marian, T. Lappi, $\mathrm{R}$. Venugopalan, The ridge in proton-proton collisions at the LHC, Phys. Lett. B697 (2011) 21-25. arXiv:1009.5295, doi:10.1016/j.physletb.2011.01.024.

[29] A. Kovner, M. Lublinsky, Angular correlations in gluon production at high energy, Phys. Rev. D83 (2011) 034017. arXiv: 1012.3398, doi:10.1103/PhysRevD.83.034017.

[30] K. Dusling, R. Venugopalan, Azimuthal collimation of long range rapidity correlations by strong color fields in high multiplicity hadron-hadron collisions, Phys. Rev. Lett. 108 (2012) 262001. arXiv:1201.2658, doi:10.1103/PhysRevLett. 108. 262001.

[31] A. Dumitru, A. V. Giannini, Initial state angular asymmetries in high energy $\mathrm{p}+\mathrm{A}$ collisions: spontaneous breaking of rotational symmetry by a color electric field and C-odd fluctuations, Nucl. Phys. A933 (2014) 212-228. arXiv:1406.5781, doi:10.1016/j.nuclphysa.2014.10.037.

[32] A. Dumitru, V. Skokov, Anisotropy of the semiclassical gluon field of a large nucleus at high energy, Phys. Rev. D91 (2015) 074006. arXiv:1411.6630, doi:10.1103/PhysRevD.91. 074006.

[33] T. Lappi, Azimuthal harmonics of color fields in a high energy nucleus, Phys. Lett. B744 (2015) 315-319. arXiv: 1501. 05505, doi:10.1016/j.physletb.2015.04.015.

[34] B. Schenke, S. Schlichting, R. Venugopalan, Azimuthal anisotropies in $\mathrm{p}+\mathrm{Pb}$ collisions from classical Yang-Mills dynamics, Phys. Lett. B747 (2015) 76-82. arXiv:1502.01331, doi:10.1016/j.physletb.2015.05.051.

[35] T. Lappi, B. Schenke, S. Schlichting, R. Venugopalan, Tracing the origin of azimuthal gluon correlations in the color glass condensatearXiv: 1509.03499.

[36] E. Iancu, D. Triantafyllopoulos, JIMWLK evolution for multiparticle production in Langevin form, JHEP 1311 (2013) 067. arXiv: 1307.1559 , doi:10.1007/JHEP11 (2013) 067. 